\title{
Earth observation and network of in situ ground sensors for disaster management and early warning
}

\author{
V. Ferrara \\ Department of Electronic Engineering, \\ Sapienza University of Rome, Italy
}

\begin{abstract}
Early warning and disaster management needs to acquire data for implementing intervention plans. Satellite and airborne remote sensing allows for Earth observation in an integral way. Furthermore, networks of sensors at ground level are used frequently both for calibrating remote sensing and for acquiring physical quantities directly in situ on the ground. Two services must be considered: communication and detection. Their weight is different in the two situations of early warning and emergency. The satellite link allows the most remote and least accessible areas to be reached. When the communication infrastructure at ground level is destroyed, the satellite link preserves continuity. In any case, an alternative sensors network on the ground could be designed. It must not rely on a traditional communications infrastructure and network electricity supply. This leads to a device design based on energy scavenging. Consequently, each network node should be realized by meeting the design specifications of low consumption and low voltage supply. This goal can be achieved by including RF SoC founded on the communication system ZigBee. The article aims: to list the characteristics of main remote sensing; to highlight problems of communication and electrical energy in remote areas of control; to present cluster architecture of nodes network that are energetically self sufficient. Keywords: remote sensing, WSN, low power, low voltage, ZigBee.
\end{abstract}

\section{Introduction}

Remote sensing has been applied more and more for different monitoring activities in the last few years. Either as satellite imaging or aerial photos, remote 
sensing is fundamental for tracking the impact of man-made or natural disasters. Moreover, they are included in health monitoring, such as syndromic surveillance for the early warning of diseases. In fact, environmental change can cause increments of epidemiology risk. Consequently the detection and monitoring of climate parameters could be correlated to the population change of disease hosts. For example, recent scientific articles underline the role of space technologies when coherent maps of vulnerability and risk of malaria are created, by using Earth Observation (EO) products and Geographic Information Systems (GIS), Anderson et al. [1]. Again, some important projects determine how satellite services can be integrated into civil protection and healthcare systems authorities, like the SAFE project of the European Space Agency (ESA) together with the World Health Organization (WHO).

However, in the monitoring and management of man-made or natural disasters, networks of sensors at level ground are frequently used. Widespread acquisition by means of seed sensors on the ground; sensors that are localized in dangerous places; monitoring of data, critical and precursory, detected more conveniently in situ on the ground: all these sensors constitute the nodes of a network and are essential for early warning and forecast activities. Together with a network, sensors must be in an operational communication infrastructure, which interconnects the field units with command centres, by sending data, pictures, video and voice exchange.

The transmission of data in real time, from in situ on the ground at operative central, uses many different systems, both fixed and mobile, wireless or not. The standard of communication can be: LAN (Local Area Network), WLAN (Wireless Local Area Network - IEEE 802.11), MAN (Metropolitan Area Network) as WiMAX (Worldwide Interoperability for Microwave Access IEEE 802.16), GPRS service, etc.

Early warning activities operate under regular conditions of normal communications. In contrast, when the effect of disaster is relevant, communication infrastructures can collapse. In effect, communication blackout involves entire regions if a serious event damages or destroys the terrestrial network. But, even if part of the infrastructure is operational, communications are difficult or impossible because traffic volume rises to high levels, making the lines quickly unavailable. Recent events, such as the earthquake in the Abruzzo province (Italy), have shown that radio amateurs are a first relevant component of responders/communicators. So, an analogical communication system should always be included in the building up of first links during the phase of emergency.

In some cases, civil protection and/or military structures do not use traditional communication support, but each subject develops or relies on a network based on: communication by dedicated satellite, together with special equipment, which is transportable and easy to configure. The satellite link allows the remotest and least accessible areas to be reached. When a communications infrastructure at ground level is destroyed, the satellite link preserves continuity, bi-directional transmission, and execution of applications including data, voice and video. 
In any case, an alternative sensors network at ground level could be designed. It must not rely on a traditional communications infrastructure and network electricity supply as the last ones are the weak nodes. This leads to a design based on energy harvesting /energy scavenging. Many examples of energy scavenging for mobile and wireless electronics have been developed in recent years, Paradiso and Starner [2]. Each is founded on one or more energy transformations: thermoelectric conversion, vibrational excitation, photovoltaic effect, etc. The progressive high integration of the circuits by the reduction of dimensional scale (nanometres), decrease of power dissipation, low voltage operating and routing optimization allows self-sufficient devices and systems to be designed, that are hardy to the effects of a catastrophic event. At present, the maximum distance between network nodes constitutes the true limit of these technologies in evolution.

Maximum and minimum distance of nodes (the distance between locations of sensors at ground level) define the range of spatial resolution for the irregular grid of network. The reduction of minimum distance raises the cost of structure realization, owing to the increase in number of sensors. Its value must be calculated by: energetic autonomy of detection station; nature of quantities under observation. The maximum distance depends on: an effective link between nodes and the central unit or hub; accuracy of analytical models for the event forecast and management.

Elliptical orbits of satellites for remote sensing are covered in a rotation time that depends on the altitude. This length of time between observations of the same area is the temporal resolution of satellite sensor. When the temporal resolution amounts to many hours or days, it limits the use of remote sensing. This is the case for emergency management. Other characteristics of satellite sensors define: spatial resolution, swath width, spectral resolution and radiometric resolution. The spatial resolution is the amount of area covered by each pixel of picture. Swath width is the ground area covered by a single overpass of the sensor. Spectral resolution is the range of the electromagnetic spectrum that is sensed. Radiometric resolution is the sensitivity for recording variations in the electromagnetic spectrum and it is measured in numbers of bits.

Different from remote sensing, an autonomous ground network preserves continuity of monitoring. Obviously, if the sensor location is not involved in the disaster area, destroyed or out of order. In any case the integration of an autonomous ground network with satellite communication augments monitoring availability and operational tools.

The most complete and integrated monitoring system includes GIS that correlates a database of all the detections, as satellite imaging, aerial photos, airborne hyper spectral remote sensing and in situ sensors. GIS must be oriented to enable: source data correction, elaboration for derivative data generation, correlation of data from different origins, etc.

Specifically, pictures of remote sensing are geo-referred and corrected to resolve: alignment problems, contrasts equalization, compensation of the effects of high frequency attenuation. Moreover, the retro-diffused energy coming from the atmosphere towards the sensor can be important for a satellite or airborne 
sensor; consequently these effects of atmosphere are corrected by using radiometric calibration on the ground, such as MIDAS (Multispectral Interactive Data Processing and Analysis System), Ferrara [3], Atturo et al. [4].

\section{Satellite sensors}

Remote sensing and communications are fundamental in all early warning activities and management of risk, emergencies and disasters.

Satellites can solve many problems of connection between inaccessible places on the ground. In fact, a communication system based on satellite assures connections between field teams, in a geographically remote location, directly and independently of the terrestrial infrastructures.

Regarding monitoring of large areas, remote sensing by satellite is convenient for an integral observation that interprets events. But characteristics as temporal resolution limit their usage when specific activity requires operational continuity.

Table 1 defines the names of main bands and table 2 lists some characteristics of the selected satellite sensors. Landsat mission uses Multispectral Scanner MSS and Thematic Mapper (Landsat 5) or Enhansed Thematic Mapper Plus ETM+ (Landsat 7), with applications on: thematic cartography and terrestrial resources.

Sensors of SPOT mission are: HRVIR (SPOT 4), High Resolution Geometric HRG and one High Resolution Stereoscopic HRS (SPOT 5). Their applications are: thematic cartography, digital topography and terrestrial resources.

The NOAA mission includes more sensors, such as: AVHRR (Advanced Very High Resolution Radiometer), Tiros Operational Vertical Sounder TOV (HIRS/2, SSU, MSU) and ATOV (HIRS/2 e AMSU). Low spatial resolution and the type of sensor assign applications of missions in the fields of: meteorology, climatology, oceanography and the low-resolution classification of vegetation.

METEOSAT and $\mathrm{M} 2 \mathrm{G}$ are used for operational meteorology, climatology and oceanography and they show high temporal resolution. Both METEOSAT and $\mathrm{M} 2 \mathrm{G}$ allow a quantitative analysis of a large area of the Earth's surface: from $65^{\circ} \mathrm{S}$ to $65^{\circ} \mathrm{N}$ parallel.

Table 2 also includes high-resolution satellite sensors, such as IKONOS and Quick Bird. They have been profitably used by ESA in seismic effect management, flood effect evaluation and emergency plans.

Table 1: $\quad$ Spectral range of bands.

\begin{tabular}{|l|c|}
\hline \multicolumn{1}{|c|}{ Band } & Spectral range ( $\boldsymbol{\mu m}$ ) \\
\hline Ultraviolet (UV) & $0.003-0.4$ \\
\hline Visible (VIS) & $0.4-0.7$ \\
\hline Infrared (IR) & $0.7-300$ \\
\hline Near Infrared (NIR) & $0.7-1.3$ \\
\hline Medium Infrared (MIR) & $1.7-4.2$ \\
\hline Thermal Infrared (ThIR) & $8-14$ \\
\hline Far Infrared & $14-1000$ \\
\hline Microwaves & $3000-3000000$ \\
\hline
\end{tabular}


Table 2: $\quad$ Characteristic of some satellite sensors.

\begin{tabular}{|c|c|c|c|c|}
\hline Sensor & $\begin{array}{c}\text { Temporal } \\
\text { resolution } \\
\text { (days) }\end{array}$ & $\begin{array}{c}\text { Spatial resolution } \\
(\mathbf{m})\end{array}$ & Band $(\mu \mathrm{m})$ & $\begin{array}{c}\text { Scene area } \\
\text { size }(\mathbf{k m})\end{array}$ \\
\hline Landsat 5 & \multirow[t]{2}{*}{16} & 30 & $\begin{array}{l}1-0.45-0.52 \\
2-0.52-0.60 \\
3-0.63-0.69 \\
4-0.76-0.90 \\
5-1.55-1.75 \\
6-10.4-12.5 \\
7-2.08-2.35 \\
\end{array}$ & \multirow[t]{2}{*}{$185 \times 185$} \\
\hline Landsat 7 & & $\begin{array}{c}30 \\
\begin{array}{l}15 \text { panchromatic } \\
\text { band }\end{array}\end{array}$ & $\begin{array}{c}1-0.45-0.52 \\
2-0.52-0.60 \\
3-0.63-0.69 \\
4-0.76-0.90 \\
5-1.55-1.75 \\
6-10.4-12.5 \\
7-2.08-2.35 \\
8-0.52-0.90 \\
\end{array}$ & \\
\hline SPOT 4 & \multirow{2}{*}{$\begin{array}{l}\text { Variable } \\
\text { (within three } \\
\text { days of } \\
\text { tasking) }\end{array}$} & 20 & \multirow{2}{*}{$\begin{array}{l}1-0.50-0.59 \\
2-0.61-0.68 \\
3-0.79-0.89 \\
4-1.58-1.73\end{array}$} & \multirow[t]{2}{*}{$56 \times 56$} \\
\hline SPOT 5 & & $\begin{array}{c}10 \\
2.5 \text { panchromatic } \\
\text { band } \\
\end{array}$ & & \\
\hline IKONOS & $\begin{array}{c}3-5 \text { off nadir } \\
144 \text { true } \\
\text { nadir }\end{array}$ & $1-4$ & $\begin{array}{l}1-0.445-0.516 \\
2-0.506-0.595 \\
3-0.632-0.698 \\
4-0.757-0.853\end{array}$ & $11 \times 11$ \\
\hline Quick Bird & $1-3.5$ & $\begin{array}{l}0.6-0.7 \text { panchrom. } \\
2.4-2.8 \text { multispectr. }\end{array}$ & $\begin{array}{c}1-0.45-0.52 \\
2-0.52-0.6 \\
3-0.63-0.69 \\
4-0.76-0.9\end{array}$ & $16.5 \times 16.5$ \\
\hline NOAA-AVHRR/3 & 0.25 & 1100 & $\begin{array}{l}1-0.58-0.68 \\
2-0.725-1.1 \\
3-3.55-3.93 \\
4-1.58-1.64 \\
5-10.3-11.3 \\
6-11.5-12.5 \\
\end{array}$ & - \\
\hline METEOSAT & $\begin{array}{c}0.0208 \\
\left(300^{\prime}\right)\end{array}$ & \multirow{2}{*}{$\begin{array}{l}2.5 \text { (VIS) } \\
5 \text { (MIR) } \\
5 \text { (ThIR) }\end{array}$} & \multirow{2}{*}{$\begin{array}{c}1-3-0.5-0.9 \\
4-5.7-7.1 \\
5-10.5-12.5\end{array}$} & \multirow[t]{2}{*}{$65^{\circ} \mathrm{S}-65^{\circ} \mathrm{N}$} \\
\hline $\mathrm{M} 2 \mathrm{G}$ & $\begin{array}{c}0.0104 \\
\left(15^{\prime}\right)\end{array}$ & & & \\
\hline
\end{tabular}

Table 3 shows characteristics of the airborne MIVIS (Multispectral Infrared and Visible Imaging Spectrometer) sensor. Like any airborne imagery, it needs a process of terrain-correction. MIVIS uses four spectrometers and 102 spectral bands with 3-4 $\mathrm{m}$ of spatial resolution. This large number of spectral bands allows the analysis of both the chemical and physical parameters of the Earth's surface. Indeed, MIVIS allows for efficient territorial classification with high spatial resolution. 
Table 3: $\quad$ Airborne MIVIS sensor characteristics.

\begin{tabular}{|c|c|c|c|c|}
\hline $\begin{array}{c}\text { Spatial } \\
\text { resolution (m) }\end{array}$ & Spectrometers & $\begin{array}{c}\text { Number of } \\
\text { Bands }\end{array}$ & $\begin{array}{c}\text { Spectral range } \\
(\boldsymbol{\mu} \mathbf{m})\end{array}$ & $\begin{array}{c}\text { Bandwidths } \\
(\boldsymbol{\mu m})\end{array}$ \\
\hline \multirow{3}{*}{4} & I & 20 & $0.43-0.83$ & 0.02 \\
\cline { 2 - 5 } & II & 8 & $1.15-1.55$ & 0.06 \\
\cline { 2 - 5 } & III & 64 & $2.0-2.5$ & 0.009 \\
\cline { 2 - 5 } & IV & 10 & $8.2-12.7$ & $0.34-0.54$ \\
\hline
\end{tabular}

There are many examples of airborne sensors whose employment is in an emergency state. But normally, they give technical answers to operators for the analysis of damage after a disaster: the disaster assessment, Clark et al. [5].

Costs, delivery wait, difficulty in obtaining a personalized detection area are different for each mission. Both the long revisit time of Landsat and decrease of useful area of imagery due to clouds can reduce its adoption in the case of emergency management. The disadvantages of using SPOT are: high cost for remote sensing in a personalized area, availability of only four bands (three in the visible and one in thermal infrared), masking by clouds.

Other sensors operate at the microwave band, i.e., operating at a frequency which is not attenuated significantly by clouds. In contract with previous sensors, they are satellites working in active ways, with on-board SAR (Synthetic Aperture Radar) and a spatial resolution of 3-100 m. This is the case of: RADARSAT-1, ERS-1 (European Remote Sensing satellite), ERS-2, ASARENVISAT (Advanced Synthetic Aperture Radar) missions. These sensors allow the evaluation of damages after a catastrophic event as earthquake or river flood by means of interferometric coherence.

\subsection{SAR application examples: river flood and landslide}

In order to evaluate hydrologic water balance, some parameters, such as runoff, infiltration and evaporate transpiration are extracted by radar remote sensing, Schultz [6]. In the same mode, the extension of the flooding areas can be measured and localized evaluating soil moisture. Unfortunately, the value of the latter could be wrong because the state of vegetation and roughness influence it. This is truer at C-band (4-8 GHz). By decreasing frequency at L-band (1$2 \mathrm{GHz}$ ), with an electromagnetic signal frequency lower $1.4 \mathrm{GHz}$, these influences are less significant. Specifically, the main parameter measured by means of SAR at L-band is the brilliance temperature. From it, soil moisture rises too.

In order to evaluate soil deformation, a similar differential interferometry method, based on SAR, can be adopted. The measure of phase difference between two SAR images of the same area can assure strain measurement with around one millimetre of precision. But the two images are realized at different times and from different points of view normally. So, the topography influences the evaluation by adding a relative phase variation that must be compensated. Atmospheric conditions have further effects on the phase. Compensation of these 
effects is obtained by means of Permanent Scatterers, i.e., radar targets that are uninfluenced.

\section{In situ ground sensors}

Examples of SAR applications have shown how remote sensing produces information of territorial areas by indirect way normally. In the same way, the classification of elements at ground level that bases its methodology on spectral signature. In order to classify a whole area, some training areas are used for calibrating detection methods. That is, it needs a check in predetermined situ, by measuring physical quantities directly. Moreover, temporal resolution of satellite sensors can be insufficient for continued control of the area of interest. So, a number of discrete sensors at ground level should be activated as instruments of calibration for remote sensing or as nodes of an alternative measurement system based on sensors network. Each sensor is specialized for the type of quantity checked. In order to correlate different quantities, frequently a complex system in situ organizes more sensors for multiple, synchronous and asynchronous detections. Data collected by each system node should be transmitted to operative central which is far away from it. The power supply can be problematic if this system has been built in place which is accessible with difficulty. These two aspects, communication link and power supply, together with cost can limit the number of systems that are designed.

In recent years, technological progress has increased the integration to a high scale and decreased power consumption of the electronic components to lower levels. For example, more tests can be carried out from few blood drops by means of very small lab-on-chip: an integrated laboratory for clinical tests.

The design of the device with the characteristics of low-voltage and lowpower, allows one to search for innovative ways for increasing its autonomy from the point of view of energy. Reduction in consumption is a target of modern electronic systems based on the principle of "Ambient Intelligence" [7]. From the point of view of architecture, low-power performances of a network can be obtained by improving routing and software management based on algorithm as "Ad-hoc On-demand Distance Vector". Obviously, characteristics, such as large distances between nodes and high measurement rate, increase power demand. But network structure can be realized by minimizing nodes that require more power and are localized inside specific places: where electrical the connection can be assured. Generally, low-power measurement nodes, that are spread in a local area, organize a cluster. Nodes are connected to each other directly and to another cluster. Only if distance is important or there is no line of sight, link is by a transmitter of higher power.

So, when it is necessary to organize a lot of measurement points inside a local area, wireless sensors network (WSN) fault-tolerance, energetically effective and low cost could be founded on ZigBee systems, Akyildiz et al. [8, 9]. 


\subsection{ZigBee}

IEEE 802.15.4 is a wireless radio frequency standard for low-power and short range applications: specific for Low-Rate Wireless Personal Area Network (WPAN), IEEE std. [10, 11]. ZigBee is a communication system, a network layer on top of the IEEE 802.15.4 standard, including PHYsical (PHY) and Message Authentication Code (MAC) layers. It offers mesh networking as interoperability between different vendors' products.

Transmission bands are $2.4 \mathrm{GHz}, 915 \mathrm{MHz}$ and $868 \mathrm{MHz}$. Modulation is BiPhase Shift Keying (BPSK) for 868 and $915 \mathrm{MHz}$ bands and Offset quadrature phase-shift keying (OQPSK) for $2.4 \mathrm{GHz}$. Access mode is the Carrier Sense Multiple Access / Collision Avoidance (CSMA/CA): nodes verify if a channel is free before transmission. But when transmission needs a low latency, access mode is the Guaranteed Time Slots (GTS).

Typical maximum power of transmission is $0 \mathrm{dBm}$, but transceivers can operate with a higher value (e.g., $15 \mathrm{dBm}$ for Embit EMB-Z1321). Second generation ZigBee/ IEEE 802.15.4 RF system-on-chip, such as CC2520 of Texas Instruments includes a transceiver whose current absorption is equal to $33.6 \mathrm{~mA}$, $25.8 \mathrm{~mA}$ and less $1 \mu \mathrm{A}$ respectively for $5 \mathrm{dBm}, 0 \mathrm{dBm}$ transmission power and in down power. Typical receiver sensitivity of the same TI's transceiver is $-98 \mathrm{dBm}$ and its current absorption at $-50 \mathrm{dBm}$ is $18.5 \mathrm{~mA}$. The link budget $(103 \mathrm{~dB}=5 \mathrm{dBm} \mathrm{Tx}+98 \mathrm{dBm} \mathrm{Rx})$ allows about $400 \mathrm{~m}$ line of sight range. Lowpower consumption of $\mathrm{SoC}$ springs from short transition times between operating modes.

CC2520 integrates: transceiver, micro control unity (8051 MCU), $8 \mathrm{~KB}$ of RAM and up to $256 \mathrm{~KB}$ of Flash memory. Consequently, nodes can be built with a very low total bill of-material costs, for managing many and different sensors connected to it. Alternative TIs MSP430 microcontroller for devices operating up to $25 \mathrm{MHz}$ shows $160 \mu \mathrm{A} / \mathrm{MHz}$ consumption. A so low microamper per megahertz consumption allows one to use systems based on harvesting energy, for supplying power to the node network.

Video-surveillance of a structure, like an airport, is an example of WSN, even with thousands of nodes, that can be designed using ZigBee systems and harvesting energy.

\subsection{Energy harvesting}

A dense deployment of sensors on the territory could be necessary if the management tools or simulation models require high spatial resolution.

For example, a hydraulic one-dimensional model requires detection of transversal sections for characterizing a riverbed. The maximum distance between two sections and resolution of the topography can depend on many criteria, also subjective, Cunge et al. [12], Bates [13].

The sustainability of network design which includes a lot of wireless nodes is energetic type primarily. Many research teams investigated more energy conversion such as: thermoelectric, vibrational excitation, photovoltaic, RF, radiation, pressure variation, Ammar et al. [16], Ottmann et al. [17]. Many 
recent sensors need low electrical power on average. For example, a typical wireless temperature combined with pressure sensor for automotive application requires about $3.8 \mu \mathrm{W}$ of electrical power, Schmidt [15].

If the transmission power of a transceiver is $0 \mathrm{dBm}(1 \mathrm{~mW})$, the power peak rises around $3 \mathrm{~mW}$ which adds power consumption of peripheral devices and circuitry for a total power consumption at around 4-5 $\mathrm{mW}$. Average power demand of transceivers is about $100 \mu \mathrm{W}$, by considering a low rate $(1 \%)$ of peak transmission. So, systems must include an energy storage device in addition to a power converter, for supplying demanded power peaks, Ammar et al. [16]. Comparison among different power sources, Roundy et al. [14], demonstrates the sustainability of Micro Electro Mechanical System (MEMS) energy converters.

In particular, Texas Instruments completes SoC CC2520/MSP430 with a maker of miniature piezoelectric actuators, named AdaptivEnergy/Joule-Thief, which collects and stores energy from tiny mechanical vibrations.

\section{Conclusions}

Earth observation by a network of sensors in situ on the ground is evolving to low-power WSN. This allows one to design the network with a high number of sensor nodes that are self sufficient from the point of view of power supply by means of the use of energy converters. The "energy harvesting" theme is research that assumes more importance with the decrease of power consumption of electronic devices and $\mathrm{SoC}$ in general.

Communication continuity, at least in one direction, can be resolved by using the new generation of the ZigBee system also. Synergy of remote sensing and WSN in situ on the ground is desirable in any case, for getting together: integral data of Earth observation by remote sensing and discrete measures, localized by WSN.

\section{References}

[1] Anderson, E., Girard, T., Ottavianelli, G., A micro-satellite and in situ ground sensor network for combating malaria, 54th International Astronautical Congress of the International Astronautical Federation, the International Academy of Astronautics, and the International Institute of Space Law, Bremen, Germany, 2003

[2] Paradiso, J. A., Starner, T., Energy scavenging for mobile and wireless electronics, IEEE CS and IEEE ComSoc, Pervasive computing, pp. 18-27, 2005

[3] Ferrara, V, Brownfield identification: different approaches for analysing data detected by means of remote sensing, Proceedings of International Conference Brownfields 2008, ed. Brebbia, Wit Press Southampton, pp. 4554, 2008

[4] Atturo, C., Cianfrone, C., Ferrara, V., Fiumi, L., Fontinovo, G., Ottavi, C.M., Remote Sensing Detection Techniques for Brownfield Identification 
and Monitoring by GIS Tools, Proceedings of 3rd International Conference Brownfields III, Ed. Brebbia, Wit Press Southampton, pp. 241-250, 2006

[5] Clark, J., Parsons, A., Zajkowski, T., Lannom, K., Remote Sensing Imagery Support for Burned Area Emergency Response Teams on 2003 Southern California Wildfires, RSAC-2003-RPT1, 2003

[6] Schultz, G.A., Meso-scale modelling of runoff and water balances using remote sensing and other GIS data, Hydrol. Science Journal, 39, 2, 1994.

[7] ISTAG Report, Ambient intelligence: from vision to reality, $\mathrm{ftp}: / / \mathrm{ftp}$.cordis.europa.eu/pub/ist/docs/istagist2003_consolidated_report.pdf

[8] Akyildiz, F. et al., A survey on sensor networks. IEEE Comm. Mag., 40(8), pp.102-114, 2002.

[9] Akyildiz, F. et al., A survey on wireless multimedia sensor networks, Computer Networks, Vol. 51, pp. 921-960, 2007

[10] IEEE std. 802.15.4 - 2003: Wireless Medium Access Control (MAC) and Physical Layer (PHY) specifications for Low Rate Wireless Personal Area Networks (LR-WPANs) http://standards.ieee.org/getieee802/download/ 802.15.4-2003.pdf

[11] IEEE std. 802.15.4 - 2006: Wireless Medium Access Control (MAC) and Physical Layer (PHY) specifications for Low Rate Wireless Personal Area Networks (LR-WPANs) http://standards.ieee.org/getieee802/download/ 802.15.4-2006.pdf

[12] Cunge, J.A., Holly, F.M., Vernwey, A., Practical aspects of Computational River Hydraulics, Pitman, London, 1980

[13] Bates, P.D., Computationally efficient modelling of flood inundation extent, European Science Foundation Workshop, Bologna (Italy), Ed. BIOS, pp. 285-301, 2004

[14] Roundy, J.S., Leland, E.S., Baker, J., Carleton, E., Reilly, E., Lai, E., Improving power output for vibration-based energy scavengers, Pervasive Comput. 4, pp. 28-36, 2005

[15] Schmidt, F., Wartungsfreie Funksensoren für Gebäudetechnik, Industrie und Automobiltechnik, in Proceedings of the Fifth Wireless Technologies Congress, Sindelfingen, Germany, 2003.

[16] Ammar, Y., Buhrig, A., Marzencki, M., Charlot, B., Basrour, S., Matou, K., Renaudin, M., Wireless sensor network node with asynchronous architecture and vibration harvesting micropower generator, in Proceedings of the Oc-EUSAI Conference, Grenoble, France, 2005.

[17] Ottmann, G., Hofmann, H., Bhatt, A., Lesieutre, G., Adaptive piezoelectric energy harvesting circuit for wireless remote power supply, IEEE Trans. Power Electron.17, pp. 669-676, 2002 